\title{
Recurrence after ESD curative resection for early gastric cancer
}

\author{
Ayako Kamiya ${ }^{1}$, Hitoshi Katai ${ }^{*}$, Kenichi Ishizu' ${ }^{1}$ Takeyuki Wada ${ }^{1}$, Tsutomu Hayashi', Sho Otsuki ${ }^{1}$, \\ Yukinori Yamagata', Takaki Yoshikawa', Shigeki Sekine², Tomohiko Nishi ${ }^{3}$, Yuka Kawasaki ${ }^{4}$, Takafumi Ito ${ }^{4}$ \\ and Hideharu Domoto ${ }^{5}$
}

\begin{abstract}
Background: Endoscopic submucosal dissection (ESD) is gaining ground as a minimally invasive treatment for early gastric cancer (EGC) that has a negligible risk of lymph node metastasis. According to the 5 th edition of Japanese gastric cancer treatment guidelines, annual or biannual follow-up with endoscopy is recommended, but follow-up with abdominal ultrasonography or computed tomography (CT) for surveillance of metastases is not recommended after the eCuraA resection. However, we experienced a case of lymph node recurrence following ESD resulting in eCuraA.

Case presentation: A 76-year-old female received ESD for EGC in a previous hospital 4 years ago. Pathological findings were tub1, $30 \mathrm{~mm}$, T1a (M), ULO, Ly0, V0, pHM-, pVM- (eCuraA) according to the 15th edition of Japanese Classification of Gastric Carcinoma. Follow-up esophagogastroduodenoscopy revealed submucosal tumor, which was suspected as a swollen lymph node by $\mathrm{CT}$ and endoscopic ultrasound fine-needle aspiration revealed the recurrence of gastric cancer. We performed total gastrectomy with D2 lymph node dissection. Postoperative pathological examination revealed no local recurrent tumor at the ESD site in the stomach. Swollen lymph node was diagnosed as metastasis and lymph node metastasis was limited near the cardia.
\end{abstract}

Conclusion: This case provides valuable information about tumor with a minimum poorly differentiated adenocarcinoma component may develop lymph node metastasis even satisfying the guidelines criteria for curative resection.

Keywords: Gastric cancer, Endoscopic submucosal dissection, Neoplasm metastasis, Recurrence, Gastrectomy

\section{Background}

Endoscopic submucosal dissection (ESD) is gaining ground as a minimally invasive treatment for early gastric cancer (EGC) with a negligible risk of lymph node metastasis [1]. The pathological criteria of endoscopic treatment based on retrospective examinations of surgical resection cases are feasible. However, we experienced a case of lymph node recurrence following ESD resulting in a pathologically curative resection of EGC.

\footnotetext{
${ }^{*}$ Correspondence: hkatai@ncc.go.jp

1 Department of Gastric Surgery, National Cancer Center Hospital, 5-1-1

Tsukiji, Chuo-ku, Tokyo 104-0045, Japan

Full list of author information is available at the end of the article
}

\section{Case presentation}

A 76-year-old female received ESD for EGC in the previous hospital 4 years ago. Pathological findings were tub1, $30 \mathrm{~mm}, \mathrm{~T} 1 \mathrm{a}(\mathrm{M})$, UL0, Ly0, V0, pHM-, pVM- according to the 15th edition of Japanese Classification of Gastric Carcinoma [2], therefore curative resection was achieved (eCuraA). She was followed up by annual esophagogastroduodenoscopy (EGD), which indicated no evidence of metastasis in 3 years. During follow-up submucosal tumor was detected in EGD, it was suspected as a swollen lymph node in computed tomography (CT) image and endoscopic ultrasound fine-needle aspiration showed recurrence of gastric cancer. She was referred to our hospital.

There were no abnormalities in the physical examination. Laboratory studies showed within normal 
range. Serum CA125 level was $278 \mathrm{ng} / \mathrm{mL}$ (normal range, $<35 \mathrm{ng} / \mathrm{mL}$ ), and other serum tumor markers were normal, including CEA, CA19-9 and $\alpha$-fetoprotein. She received low anterior resection for rectal cancer 10 years ago, and pathological findings were tub1, T3(SS), Ly1, V1, INF b, int, pPM-, pDM-, pN0, fM0, fStage II according to the 3rd English edition of Japanese Classification of Colorectal, Appendiceal, and Anal Carcinoma. She also received partial hepatectomy for liver metastasis and upper right lobe partial excision for lung metastasis 9 years ago.

EGD found ESD scar at the lesser curvature of antrum (Fig. 1a), and a submucosal tumor at the lesser curvature of cardia (Fig. 1b). CT showed an approximately $30-\mathrm{mm}$, high density, round mass at the lesser curvature of upper gastric body (Fig. 1c). There were neither ascites nor other evidences of metastasis. Uptake of fluorodeoxyglucose was seen on positron emission tomography-CT (Fig. 1d). Therefore, lymph node metastasis was diagnosed as resulting from the EGC originally treated by ESD.
Total gastrectomy with D2 lymph node dissection was carried out. Because tumor invaded distal part of the pancreas, distal pancreatectomy was added. The total operating time was $337 \mathrm{~min}$. The intraoperative blood loss was $647 \mathrm{~mL}$. Pancreatic fistula and intra-abdominal abscess (Clavien-Dindo classification: Grade 3a) occurred after surgery, and she was discharged on 74 days of operation.

The resected specimen had the submucosal tumor with ulceration on the top, which had not been observed by EGD before surgery. The tumor measured $37 \times 33 \mathrm{~mm}$ (Fig. 2b). Postoperative pathological examination revealed no local recurrent tumor at the ESD site in the stomach. Poorly differentiated adenocarcinoma occupied the majority of the tumor and metastasis was limited to the mural tumor considered to be a lymph node near the cardia (Fig. 2c). We thought that the EGC resected by ESD was the origin of the lymph node metastasis because the swollen lymph node was a regional lymph node, there was no other primary tumor, and their pathological characters are similar. The status of HER2 expression was negative. No metastasis was observed in other lymph nodes retrieved at the surgery.

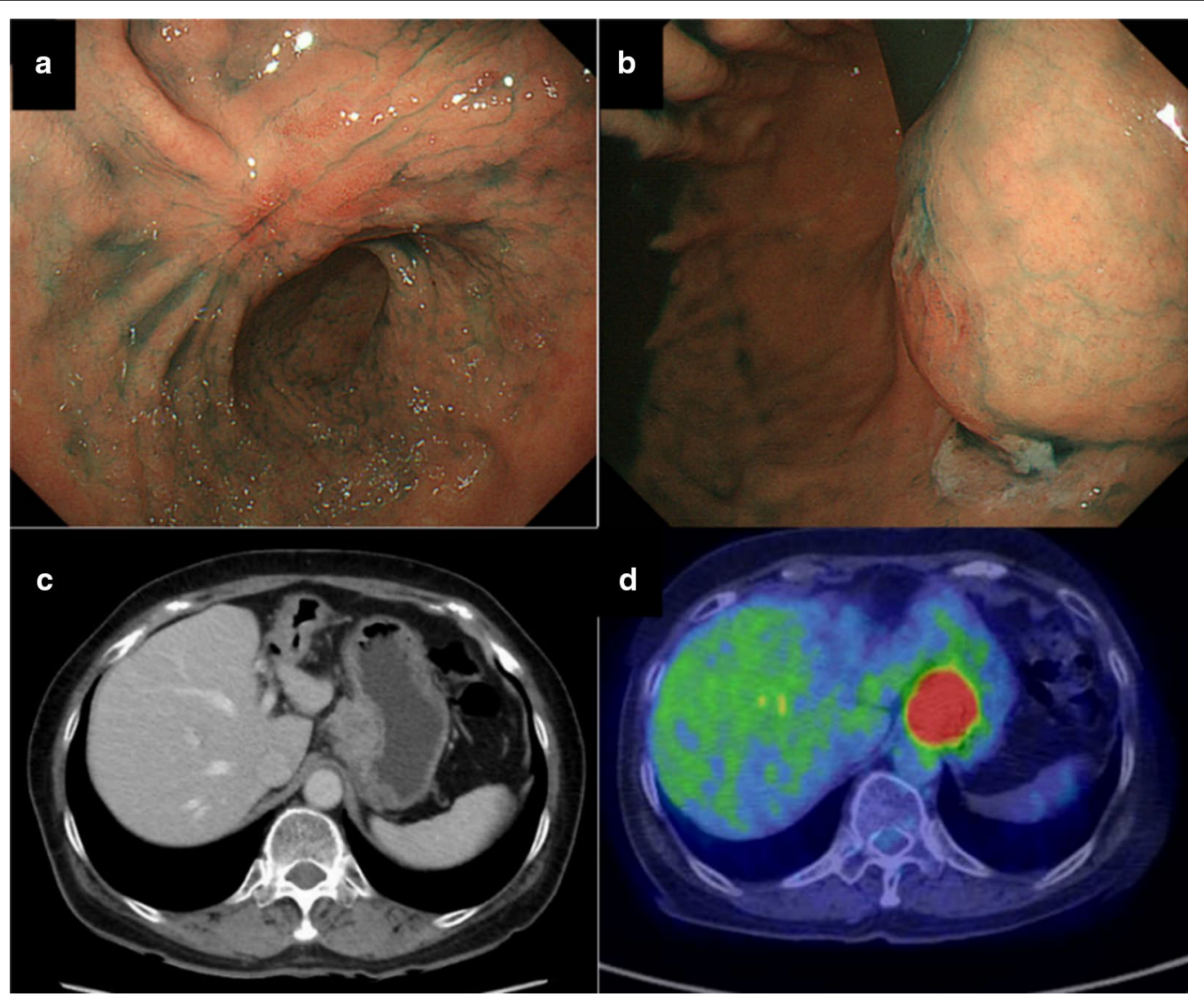

Fig. 1 Preoperative findings. a, b EGD found ESD scar at the lesser curvature of antrum and a SMT at the lesser curvature of cardia. c CT shows an approximately 30-mm, high density, round mass at the lesser curvature of upper gastric body. $\mathbf{d}$ Uptake of fluorodeoxyglucose seen on PET-CT 


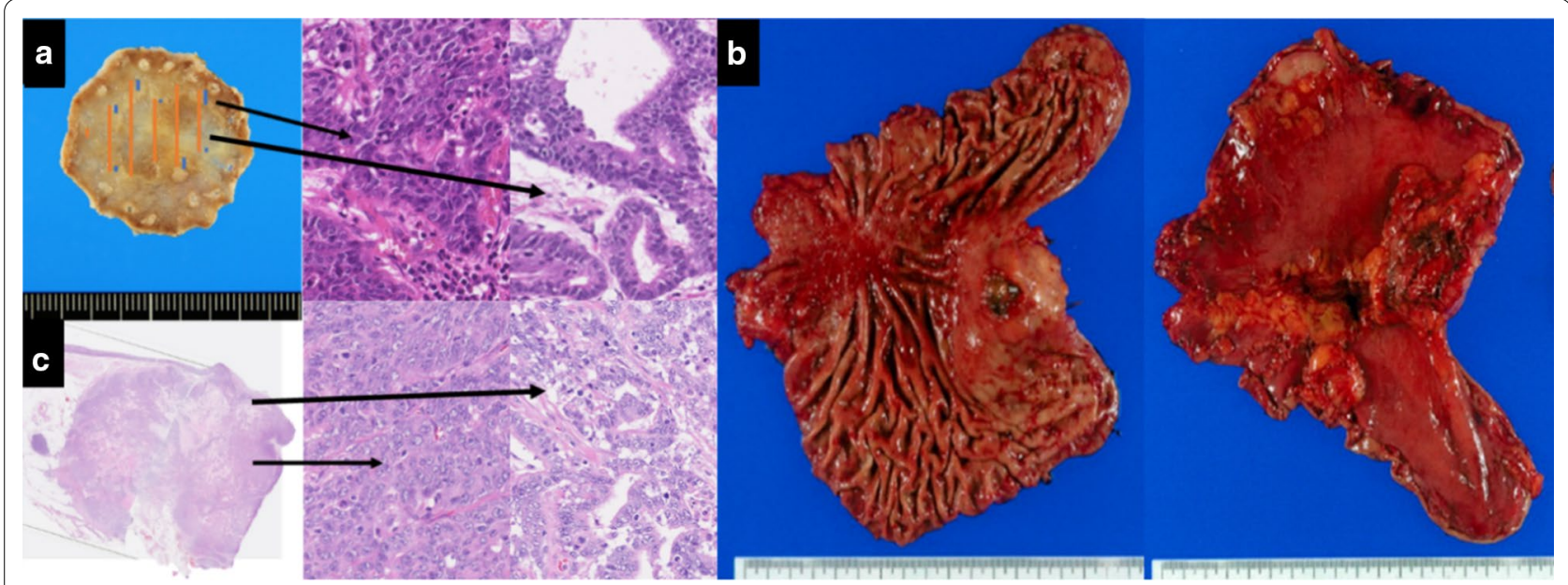

Fig. 2 Pathological findings. a ESD specimen predominantly reveals a well to moderately differentiated adenocarcinoma with a poorly differentiated component which the blue lines indicate. The orange lines indicate the cancer. $\mathbf{b}$ The tumor measures $37 \times 33 \mathrm{~mm}$. c A lymph node resected by surgery reveals a moderately to poorly differentiated adenocarcinoma

She has received adjuvant chemotherapy with S-1. No recurrence has been subsequently detected 9 months after the surgery.

\section{Discussion}

According to the 5th edition of Japanese gastric cancer treatment guidelines, the resection is classified as endoscopic curability A (eCuraA) when all of the following conditions are fulfilled, provided the cancer is without ulcerative findings (ULO) regardless of tumor size, or with ulcerative findings (UL1) less than $3 \mathrm{~cm}$ in size: en bloc resection, any tumor size, histologically differentiated type-dominant, pT1a, negative horizontal margin (HM0), negative vertical margin (VM0) and no lymphovascular [3]. This case was diagnosed as eCuraA for ESD.

Gotoda et al. and Hirasawa et al. reported that no surgical EGC cases without lymphovascular involvement had lymph node metastases in intramucosal differentiated type cancers over $2 \mathrm{~cm}$ in size without ulceration, intramucosal differentiated type cancers less than $3 \mathrm{~cm}$ in size with ulceration $[4,5]$. JCOG0607 included 470 patients with clinically diagnosed differentiated type intramucosal cancers without ulceration or less than $3 \mathrm{~cm}$ in size with ulceration, and 371 patents who had achieved curative resection had no recurrence [6]. Oda et al. reported no local recurrence and 1 metastasis in a multicenter study on long-term outcomes of eCuraA in 2723 patients with EGC [7]. This case was diagnosed as eCuraA. Follow-up with annual or biannual endoscopy is recommended after the eCuraA resection. However, follow-up with abdominal ultrasonography or CT for surveillance of metastases is not recommended after the eCuraA resection.

On this favorable trend of ESD for EGC, we experienced a case of lymph node recurrence after curative ESD for intramucosal gastric cancer. It is a rare case for we searched the core database Pubmed up to May, 2020 for the literature about the similar case using "ESD" and "eCuraA" as keywords, we found only 2 previous case reports which reported a recurrence after the eCuraA ESD resection including references in the report (Table 1) $[8,9]$.

Hanaoka et al. reported a case of nodal recurrence and liver metastasis after curative ESD of a moderately differentiated-dominant intramucosal EGC $55 \mathrm{~mm}$ in size,

Table 1 Recurrences after the eCuraA ESD resection

\begin{tabular}{|c|c|c|c|c|c|c|c|}
\hline Case & Author/year & Age/sex & Differentiation & $\begin{array}{l}\text { Size } \\
(\mathrm{mm})\end{array}$ & UL & Recurrence & $\begin{array}{l}\text { Period } \\
\text { until recurrence }\end{array}$ \\
\hline 1 & Hanaoka/2009 [8] & 60/male & Differentiated type-dominant & 55 & - & $\begin{array}{l}\text { Lymph node } \\
\text { Liver }\end{array}$ & 14 months \\
\hline 2 & Fujii/2015 [9] & 70/male & Differentiated type & 22 & + & Lymph node & 17 months \\
\hline 3 & Our case/2020 & $\begin{array}{l}76 \\
\text { /female }\end{array}$ & Differentiated type-dominant & 30 & - & Lymph node & 4 years \\
\hline
\end{tabular}


intermingled with poorly differentiated adenocarcinoma in some regions [7]. Fujii et al. reported a case of lymph node metastasis after curative ESD of a moderately differentiated intramucosal EGC, $22 \mathrm{~mm}$ in size accompanied by an ulcer [9].

The resected specimen revealed predominantly a welldifferentiated adenocarcinoma. However, a poorly differentiated adenocarcinoma component was also identified (Fig. 2a). This component might be a cause of lymph node metastasis because the resected tumor was occupied by poorly differentiated adenocarcinoma component. Lee et al. reported that lymph node metastasis rate with or without the presence of undifferentiated type histology within differentiated type tumors was $5.1 \%$ versus $0.5 \%$, respectively [10]. The presence of undifferentiated type histology within differentiated type tumors was found to be associated with lymph node metastasis in the multivariate analyses [10]. Although the guidelines described an undifferentiated component of the lesion exceeds $2 \mathrm{~cm}$ in length, the endoscopic curability is classified as $\mathrm{C}-2$ (eCuraC-2), and minimal component of undifferentiated component might be risk factor of lymph node metastasis.

In the case of Fujii et al., there was no undifferentiated component but the ulcer in the tumor [9]. The existence of ulcer is regarded as a risk factor of nodal metastasis [11]. However, Gotoda et al. reported that no surgical EGC cases out of 488 cases had lymph node metastases in intramucosal differentiated adenocarcinoma with ulcer findings less than $3 \mathrm{~cm}$ in size [4].

\section{Conclusion}

Careful follow-up is needed for patients with a poorly differentiated adenocarcinoma component, keeping in mind that metastasis may possibly occur even after curative resection based on the guideline criteria.

\section{Abbreviations}

ESD: Endoscopic submucosal dissection; EGC: Early gastric cancer; CT: Computed tomography; EGD: Esophagogastroduodenoscopy; eCura: Endoscopic curability; UL: Ulcerative findings; HM: Horizontal margin; VM: Vertical margin.
\end{abstract}

\section{Acknowledgements}

Not applicable.

\begin{abstract}
Authors' contributions
AK and $\mathrm{HK}$ conceived of the presented idea, analyzed and interpreted the patient data. YK, TI performed ESD. AK and HK performed the surgery. SS and HD diagnosed pathologically. TN made substantial contributions to the acquisition of data. AK and HK were major contributors in writing the manuscript with support from KI, TW, TH, SO, YY, TY, and SS. All authors read and approved the final manuscript.
\end{abstract}

\section{Funding}

This report was carried out without funding.

\section{Availability of data and materials}

Data sharing is not applicable to this article, since datasets were neither generated nor analyzed for the case report.

Ethics approval and consent to participate

Not applicable.

\section{Consent for publication}

Informed consent was obtained from the patient and the patient's family for the publication of this case report.

\section{Competing interests}

The authors declare that they have no competing interests.

\section{Author details}

${ }^{1}$ Department of Gastric Surgery, National Cancer Center Hospital, 5-1-1 Tsukiji, Chuo-ku, Tokyo 104-0045, Japan. ${ }^{2}$ Department of Pathology, National Cancer Center Hospital, 5-1-1 Tsukiji, Chuo-ku, Tokyo 104-0045, Japan. ${ }^{3}$ Department of Surgery, Keiyu Hospital, 3-7-3 Minatomirai, Nishi-ku, Yokohama City, Kanagawa 220-8521, Japan. ${ }^{4}$ Department of Gastroenterology, Keiyu Hospital, 3-7-3 Minatomirai, Nishi-ku, Yokohama City, Kanagawa 220-8521, Japan.

${ }^{5}$ Department of Diagnostic Pathology, Keiyu Hospital, 3-7-3 Minatomirai, Nishi-ku, Yokohama City, Kanagawa 220-8521, Japan.

Received: 16 October 2020 Accepted: 30 November 2020

Published online: 06 January 2021

\section{References}

1. Oda I, Saito D, Tada M, et al. A multicenter retrospective study of endoscopic resection for early gastric cancer. Gastric Cancer. 2006;9:262-70.

2. Japanese Gastric Cancer Association. Japanese Classification of Gastric Carcinoma. 15th ed. Tokyo: Kanehara; 2017.

3. Japanese Gastric Cancer Association. Japanese gastric cancer treatment guidelines. 5th ed. Tokyo: Kanehara; 2018.

4. Gotoda T, Yanagisawa A, Sasako M, et al. Incidence of lymph node metastasis from early gastric cancer: estimation with a large number of cases at two large centers. Gastric Cancer. 2000;3:219-25.

5. Hirasawa T, Gotoda T, Miyata S, et al. Incidence of lymph node metastasis and the feasibility of endoscopic resection for undifferentiated-type early gastric cancer. Gastric Cancer. 2009;12:148-52.

6. Kurokawa Y, Hasuike N, Ono H, et al. Gastrointestinal Oncology Study Group of Japan Clinical Oncology Group. A phase II trial of endoscopic submucosal dissection for mucosal gastric cancer: Japan Clinical Oncology Group Study JCOG0607. J Clin Oncol. 2009:39:464-6.

7. Oda I, Oyama T, Abe $\mathrm{S}$, et al. Preliminary results of multicenter questionnaire study on long-term outcomes of curative endoscopic submucosal dissection for early gastric cancer. Dig Endosc. 2014;26:214-9.

8. Hanaoka N, Tanabe S, Higuchi K, et al. A rare case of histologically mixed type intramucosal gastric cancer accompanied by nodal recurrence and liver metastasis after endoscopic submucosal dissection. Gastrointest Endosc. 2009:69:588-90.

9. Fujii H, Ishii E, Tochitani S, et al. Lymph node metastasis after endoscopic submucosal dissection of a differentiated gastric cancer confined to the mucosa with an ulcer smaller than $30 \mathrm{~mm}$. Dig Endosc. 2015;27:159-61.

10. Lee JH, Choi IJ, Han HS, et al. Risk of lymph node metastasis in differentiated type mucosal early gastric cancer mixed with minor undifferentiated type histology. Ann Surg Oncol. 2015;22:1813-9.

11. Sekiguchi M, Kushima R, Oda I, et al. Clinical significance of a papillary adenocarcinoma component in early gastric cancer: a single-center retrospective analysis of 628 surgically resected early gastric cancers. J Gastroenterol. 2015;50(4):424-34.

\section{Publisher's Note}

Springer Nature remains neutral with regard to jurisdictional claims in published maps and institutional affiliations. 